\title{
Downregulation of microRNA-4324 promotes the EMT of esophageal squamous-cell carcinoma cells via upregulating FAK
}

This article was published in the following Dove Press journal: OncoTargets and Therapy

\author{
Jian Zhou',* \\ Jiangtao Zhu',* \\ Guojun Jiang' \\ Juncheng Feng' \\ Qianqian Wang ${ }^{2}$ \\ 'Department of Thoracic and \\ Cardiovascular Surgery, Affiliated Yixing \\ Hospital of Jiangsu University, Yixing, \\ Jiangsu Province, 214200, People's \\ Republic of China; ${ }^{2}$ Department of \\ Oncology, Affiliated Yixing Hospital of \\ Jiangsu University, Yixing, Jiangsu \\ Province, 214200, People's Republic of \\ China
}

*These authors contributed equally to this work
Correspondence: Qianqian Wang Department of Oncology, Affiliated Yixing Hospital of Jiangsu University, 75 Tongzhen Road, Yixing, Jiangsu Province, 214200, People's Republic of China Email qqianwang@yeah.net
Background: Esophageal squamous-cell carcinoma (ESCC) metastasis is the major cause of death of this severe and common malignancy. Focal adhesion kinase (FAK) is one of the key components of the focal adhesion complex, which is a multi-protein structure that controls cell adhesion, migration and invasion and regulates tumor metastasis.

Purpose: To identify the roles and mechanisms of FAK in the regulation of Epithelial-tomesenchymal transition (EMT) of ESCC cells.

Methods: The expression of FAK and miR-4324 in both ESCC tissues and cells were evaluated by qRT-PCR and Immunohistochemistry analysis. Dual luciferase assays were performed for the confirmation of miR-4324's specific binding to 3'UTR of FAK mRNA. Besides, the trans-well assays and wound healing assays were employed to evaluate the effects of FAK /miR-4324 axis on the EMT regulation of ESCC cells. Furthermore, the relationship between miR-4374/FAK expression and clinical pathologic parameters \& patient survival were also statistically analyzed.

Results: In this study, we identified the upregulation of FAK and downregulation of miR4324 in both ESCC cells and tissues. Overexpression of miR-4324 mimic, which significantly decreased cellular FAK levels, can impair the invasion potential and migration ability of ESCC cells. Besides, co-transfection of FAK can attenuate the function of miR-4324 mimic. Further experimental results demonstrated that miR-4324 mimic remarkably downregulated epithelial-to-mesenchymal transition (EMT) phenotype, which can also be effectively prevented by overexpressing FAK in ESCC cells. What's more, low miR-4324 and high FAK tissue levels have significant association with poor cell differentiation, tumor size and invasion depth as well as overall number of metastatic lymph nodes. Patients with high miR-4324 and low FAK levels in tumoral tissues lived longer than their counterparts, respectively.

Conclusions: In conclusion, miR-4324/FAK axis could be a promising therapeutic target and potential prognostic biomarker for ESCC, which deserves further investigation in the clinic.

Keywords: miRNA-4324, focal adhesion kinase, esophageal squamous-cell carcinoma, epithelial-to-mesenchymal transition, cancer progression

\section{Introduction}

Globally, Esophageal carcinoma (EC) is the ninth most common malignancy (with an age-standardized incidence rate of 6.3/100,000) and seventh leading cause of cancer mortality (with an age-standardized mortality rate of 5.5/ 100,000). ${ }^{1}$ According to estimates from the American Cancer Society (ACS), the number of new EC cases and deaths in the United States will reach 17,650 
and 16,080 in 2019 , respectively. ${ }^{2}$ People'sRepublic of China owns the world's highest incidence of EC, with Esophageal squamous-cell carcinoma (ESCC) occupying the vast majority (over $80 \%$ ). ${ }^{3}$ Despite significant advances in cancer diagnosis and treatment, the prognosis of ESCC remains poor, with a 5-year survival rate of $<20 \%{ }^{4,5}$ The high morbidity and mortality of ESCC are largely due to late diagnosis and rapid metastatic spread in the clinic. ${ }^{6}$ Most patients are firstly diagnosed with locally advanced or metastatic disease, lacking the opportunity for radical resection. ${ }^{2,7}$ In addition, the application of chemotherapy and radiotherapy is strongly limited because of the severe and often intolerable sideeffects. ${ }^{8}$ Although a variety of dysregulated molecules and potential targets of ESCC have gradually been characterized, the molecular mechanism involved in tumorigenesis and progression remains complex and largely unknown. ${ }^{9,10}$

Epithelial-to-mesenchymal transition (EMT) is a pivotal step in the process of cancer metastasis, during which non-invasive cancer cells lose epithelial phenotype and acquire invasiveness. ${ }^{11}$ Hallmarks of EMT include the dissolution of cell-cell contacts, downregulation of epithelial markers (such as E-cadherin), increased levels of mesenchymal characterizations (such as Vimentin and $\mathrm{N}$-cadherin) and induction of focal adhesion turnover, etc. ${ }^{12}$ Focal adhesion kinase (FAK) is one of the key components of the focal adhesion complex, which is a multi-protein structure that connects the extracellular matrix to the cytoskeleton of the cytoplasm, thus controlling cell adhesion, migration and invasion. ${ }^{13}$ Currently, overexpression of FAK is reported to be correlated with tumor invasiveness in several tumors, including hepatocellular carcinoma,${ }^{14}$ colorectal cancer,${ }^{15}$ leukemia,${ }^{16}$ breast cancer, ${ }^{17}$ and lung adenocarcinoma ${ }^{18}$, etc. However, evidence of FAK implication in the EMT and metastasis of ESCC is limited.

Micro RNA (miRNA) is an endogenous small noncoding RNA, which inhibits the expression of target genes by inhibiting the translation or increasing the degradation of mRNA, and plays a wide range of biological functions in cell proliferation, apoptosis, invasion, differentiation, and other important cellular processes. ${ }^{19}$ More than $30 \%$ of human genes can be regulated by various miRNAs, and it has been confirmed that abnormal miRNA expression is involved in the tumorigenesis and development of numerous malignancies. ${ }^{20,21}$
In this study, we identified that FAK, which is a direct and important biological target of miRNA-4324 (miR4324), is remarkably overexpressed in both human ESCC cells and tissues. And upregulation of miR-4324 can suppress the EMT through downregulating FAK in ESCC cells. What is more, low miR-4324 and high FAK tissue levels have significant association with poor cell differentiation, tumor size and invasion depth as well as overall number of metastatic lymph nodes. Patients with high miR-4324 and low FAK levels in tumoral tissues lived longer than their counterparts, respectively. In conclusion, miR-4324/FAK axis could be a promising therapeutic target and potential prognostic biomarker for ESCC, which deserves further investigation in the clinic.

\section{Materials and methods \\ Cells, tissues and patients}

TE-1 and Eca109 human ESCC cell lines and normal human esophageal epithelial cells (HEECs) were purchased from the American Type Culture Collection. All the cells were maintained in DMEM (Thermo Fisher Scientific, Carlsbad, USA) supplemented with 10\% FBS (Thermo Fisher Scientific, Carlsbad, USA) and maintained at $37^{\circ} \mathrm{C}$ with $5 \% \mathrm{CO}_{2}$ in an atmosphere of moist air.

Pairs of human ESCC and para-carcinoma tissues $(n=50)$ were obtained from patients who underwent radical resection of EC between January 2010 and September 2011 at Affiliated Yixing Hospital of Jiangsu University (Jiangsu, People'sRepublic of China). All patients had received no chemotherapy or radiotherapy before surgery, and signed informed consent before enrollment. The research was conducted in accordance with the Declaration of Helsinki. The Ethics Committee of Affiliated Yixing Hospital of Jiangsu University approved the whole research programme. Surgical evaluation was employed to determine the clinical stage by clinicians, while experienced pathologists used histopathologic analysis to assess the cancer type and grade.

\section{Immunohistochemistry $(\mathrm{IHC})^{22}$}

The tissue slides were deparaffinized in xylene and rehydrated in gradient alcohol. Endogenous peroxidases were eliminated by $3 \% \mathrm{H}_{2} \mathrm{O}_{2}$ at $37^{\circ} \mathrm{C}$ for 20 mins. After blocking with $1 \%$ BSA at room temperature (RT) for $1 \mathrm{hr}$, the slides were incubated with anti-FAK Rabbit mAb (1:500, \#71433, Cell 
Signaling Technology, Massachusetts, USA) at $4^{\circ} \mathrm{C}$ overnight. After being washed with PBS, the slides were incubated with horseradish peroxidase (HRP)-linked anti-rabbit IgG antibody (1:2,000, \#7074, Cell Signaling Technology, Massachusetts, USA) for $1 \mathrm{hr}$ at RT and visualized with a DAB substrate (Millipore, Darmstadt, Germany).

\section{Quantitative real-time PCR assessment}

Total tissue and cellular RNAs were isolated using Trizol Reagent (Thermo Fisher Scientific, California, USA) following the manufacturer's protocols. The isolated RNAs were then reverse transcribed into complementary DNA, equal amounts of which were employed as templates for quantitative real-time PCR (qRT-PCR) amplification (the primer sequences are listed in Table S1). The $\triangle \triangle_{C T}$ method was employed to analyze the experimental data. ${ }^{23}$

\section{Western blotting ${ }^{24}$}

Cells and tissues were lysed by a total protein extraction buffer (Beyotime Biotechnology, Shanghai, People'sRepublic of China). Equal amounts of lysate samples were then separated by SDS-PAGE and immunoblotted with primary antibodies and corresponding HRP-labeled secondary antibodies. Immuno-reactive protein bands were visualized in dark room after incubated with a DAB substrate (Millipore, Darmstadt, Germany). The primary and secondary antibodies employed in this work including anti-FAK Rabbit mAb (1:1,000, \#71433), anti-E-Cadherin Rabbit mAb (1:1000, \#3195), antiN-Cadherin Rabbit mAb (1:1,000, \#13116), anti-Vimentin Rabbit mAb (1:1000, \#5741), anti- $\beta$-Actin Rabbit mAb (1:1,000, \#4970) and (HRP)-linked anti-rabbit IgG antibody (1:2000, \#7074), which were all obtained from Cell Signaling Technology (Massachusetts, USA).

\section{Dual-luciferase report assay ${ }^{25}$}

The 3'UTR of FAK gene that was wildtype (FAK-WT) and mutated at the miR-4324 binding-site (FAK-MT) was cloned into the pGL3-basic vector. ESCC cells were grown in a 24 -well plate at approximately $80 \%$ confluence, and then transfected with both miR-4324 mimic and pGL3-FAK vector. After 24 hrs, Dual-Luciferase Reporter Assay System kit (Promega, USA) was employed to analyze the experimental results.

\section{Wound healing assays}

ESCC cells of different groups were planted into 6-well plates and grew up to $100 \%$ confluence, and a wound was then generated gently by using a $1000 \mu$ pipette tip. Then, cells were cultured in BSA-free medium for another 48 hrs. Cells in the wounded monolayer were observed and photographed at various time points, and the percent gaps were measured by Image J software (Version: 2.1.4.7). The wound healing percentage was calculated by the following equation:

Wound Healing Percentage $(\%)=\frac{\text { Percent Gaps }^{0 \mathrm{~h}}-\text { Percent Gaps }^{48 \mathrm{~h}}}{\text { Percent Gaps }^{0 \mathrm{~h}}}$

\section{Trans-well assays}

Cell migration assays were performed using 24-well Transwell plates following previous descriptions with minor modifications. ${ }^{26}$ Briefly, the under the surface of the membrane was coated with fibronectin $(10 \mathrm{mg} / \mathrm{mL})$ in $\mathrm{PBS}$ at $37^{\circ} \mathrm{C}$ for 2 hrs. The lower chamber was filled with $0.6 \mathrm{~mL}$ of DMEM supplemented with $10 \%$ FBS. Before the experiment, cells were serum-starved overnight (DMEM plus $0.5 \%$ BSA), then $1 \times 10^{6}$ cells in a volume of $0.1 \mathrm{~mL}$ migration medium (DMEM plus $0.5 \% \mathrm{BSA}$ ) were added to the upper chamber. After incubation at $37^{\circ} \mathrm{C}$ for $12 \mathrm{hrs}$, cells on the upper surface of the membrane were removed. The migrant cells attached to the lower surface were fixed in $10 \%$ formalin and stained with DAPI (10 $\mu \mathrm{g} / \mathrm{mL}$, Beyotime Biotechnology, Shanghai, People'sRepublic of China). The number of migrated cells on the lower surface of the membrane was counted under a fluorescence microscope.

\section{Statistical analysis}

The statistical analysis involving two groups was performed by umpaired Student's $t$-test, whereas ANOVA was employed to compare more than two groups. The Chisquared test was employed to examine possible correlations between the clinicopathologic parameters and miR4324/FAK expression. Kaplan-Meier methods were employed for survival analysis. All data were processed with SPSS software (Version: 16.0), and $p<0.05$ was considered statistically significant.

\section{Results}

\section{Elevated expression of FAK in ESCC} tissues and cells

In order to determine the protein levels of FAK in ESCC tissues, 50 pathologist-confirmed and clinically annotated ESCC surgical specimens were subjected to IHC staining in the present study, and the FAK expression was defined as "-,,,++++++ " according to the staining degree and area of each slide (Figure S1). The representative IHC 
A

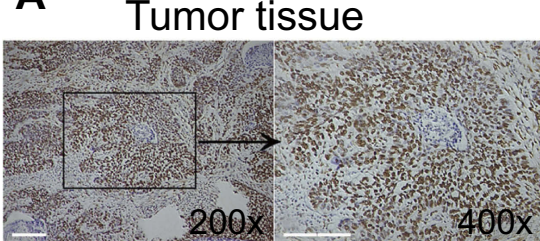

Para-carcinoma tissue

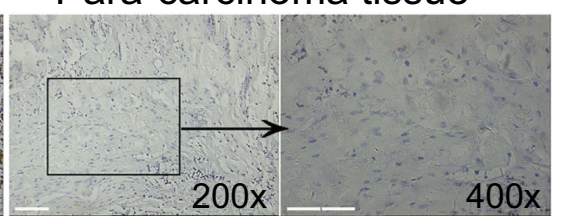

C

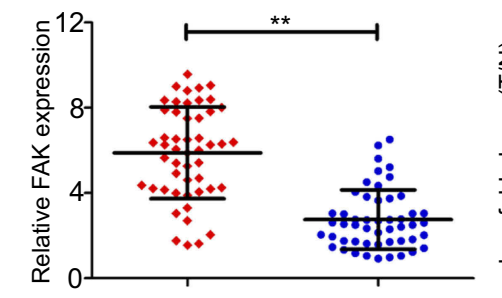

D

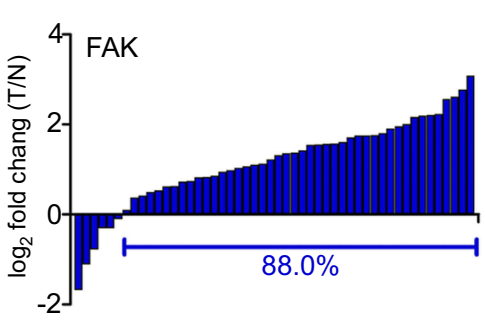

E
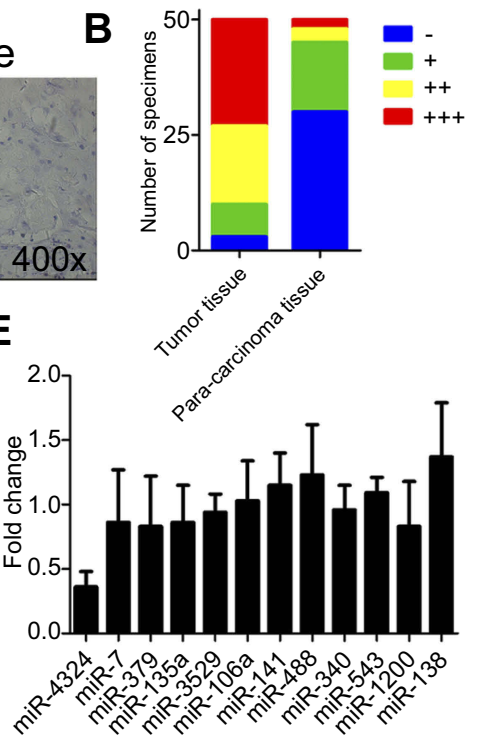

H

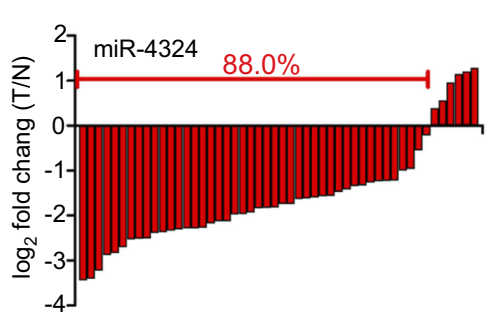

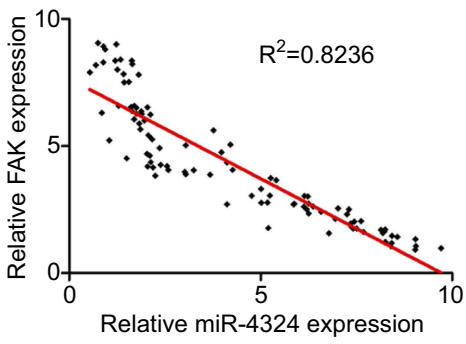

Relative miR-4324 expression
G

F

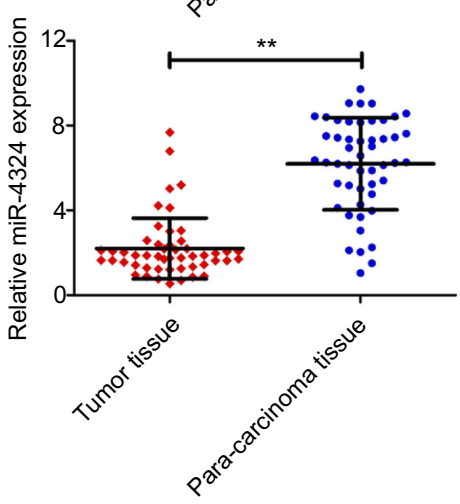

I

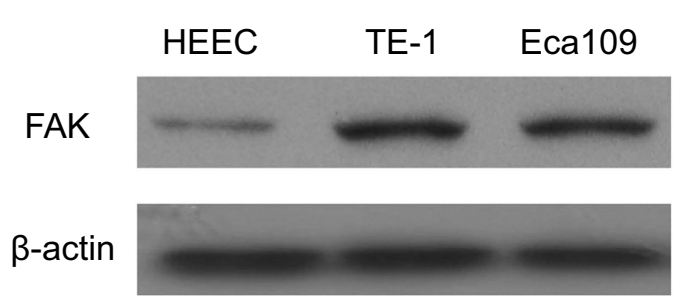

\section{J}

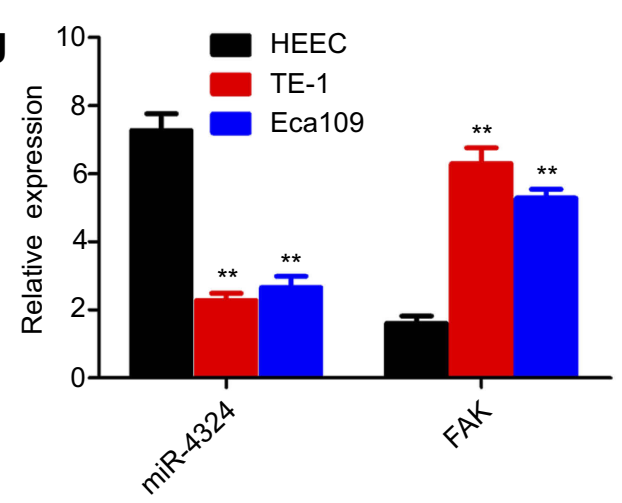

Figure I Expression of FAK and miR-4324 in ESCC tissues and cells. (A) Representative IHC photos for FAK in tumor and paired para-carcinoma tissues (magnification times: $200 \times$ and $400 \times$, Scale bars: $200 \mu \mathrm{m}$ ). (B) The number of specimens displaying different FAK levels in the group of tumor and para-carcinoma tissues. (C) Relative expression of FAK in tumor and paired para-carcinoma tissues by qRT-PCR analysis. (D) The expression of FAK for each paired sample was represented as log ${ }_{2}$ fold change of the tumor relative to para-carcinoma tissue $(\mathrm{T} / \mathrm{N})$. (E) Relative expression of different miRNAs in ESCC tumors and paired para-carcinoma tissues were determined by qRT-PCR analysis. The miRNA expression for each paired sample was represented as fold change of the tumor relative to normal para-carcinoma tissue (T/N). (F) Relative expression of miR-4324 in tumor and paired para-carcinoma tissues by qRT-PCR analysis. (G) The expression of miR-4324 for each paired sample was represented as log 2 fold change of the tumor relative to para-carcinoma tissue $(T / N)$. (H) The expression of FAK was inversely correlated with miR-4324 in ESCC tissues in all the enrolled surgical specimens. (I) Western blotting analysis for the expression of FAK in ESCC cells (TE-I and Eca 109) and normal human esophageal epithelial cells (HEEC). (J) qRTPCR analysis for the relative expression of FAK and miR-4324 in ESCC cells (TE-I and Eca 109) and normal human esophageal epithelial cells (HEEC). Data are shown as mean $\pm S D(n=3)$, *** $p<0.01$.

Abbreviations: FAK, focal adhesion kinase; ESCC, esophageal squamous cell carcinoma; IHC, Immunohistochemistry; qRT-PCR, quantitative real-time polymerase chain reaction; HEEC, human esophageal epithelial cells; SD, standard deviation.

photos of FAK in ESCC and para-carcinoma tissues were illustrated in Figure 1A, with the statistical analysis indicating that (Figure 1B) the protein levels of FAK is remarkably elevated in ESCC tissues. Consistently, qRTPCR results indicated that the average mRNA expression of FAK (overexpressed in $88 \%$ cases) is significantly 
A

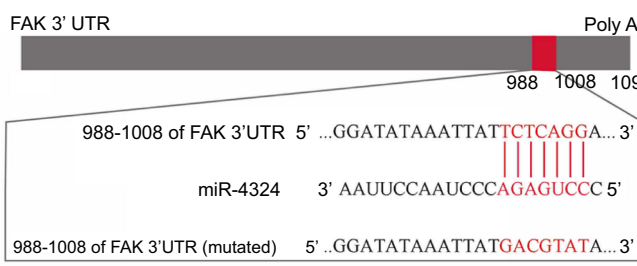

C

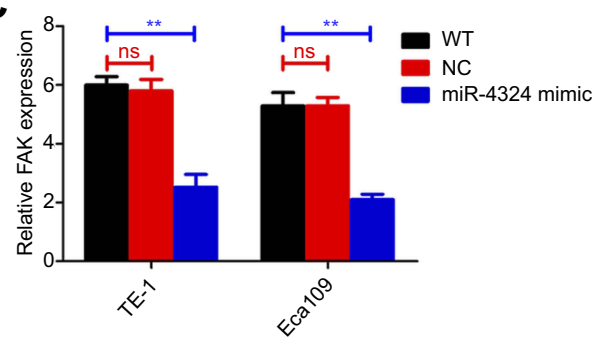

B

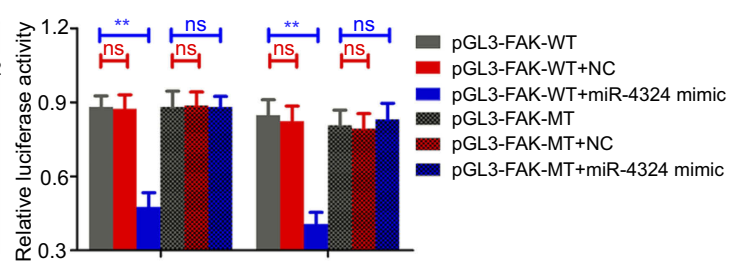

D

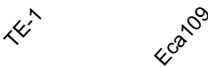

FAK

$\beta$-actin

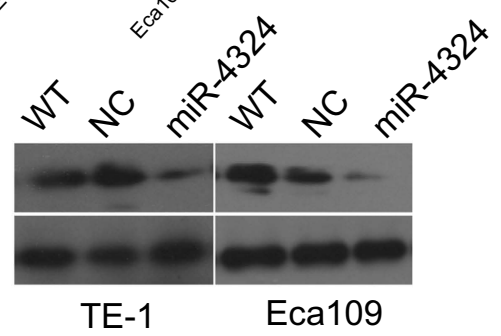

Figure 2 FAK is directly regulated by miR-4324 in ESCC cells. (A) Bioinformatics analysis demonstrates that one miR-4324 binding sites in 3'UTR of FAK. (B) Dual luciferase reporter assessment showing significant suppression of luciferase activity only when miR-4324 mimic was partnered with wild type (WT) but not mutated (MT) FAK 3'UTR in both TE-I and Eca 09 cells. (C and D) FAK can be significantly downregulated in miR-4324 mimic transfected TE-I and Eca I09 cells by qRT-PCR (C) and WB (D) analysis. Data are shown as mean $\pm \mathrm{SD}(\mathrm{n}=3), * * p<0.0 \mathrm{I}, \mathrm{ns}=$ no significance.

Abbreviations: FAK, focal adhesion kinase; ESCC, esophageal squamous cell carcinoma; UTR, untranslated regions; qRT-PCR, quantitative real-time polymerase chain reaction; WB, Western Blotting; SD, standard deviation.

increased in ESCC tissues $\left({ }^{* *} p<0.01\right.$, Figure $\left.1 \mathrm{C}-\mathrm{D}\right)$. In accordance, both the protein and mRNA levels of FAK in two ESCC cell lines (TE-1 and Eca-109) were significantly higher than that in normal HEECs (Figure 1I-J).

\section{Inverse correlation exists between miR-4324 and FAK levels in ESCC tissues}

According to the prediction of online software (http:// www.targetscan.org) and previous publications, ${ }^{27-30}$ FAK might be regulated by a wide variety of micro RNAs, such as miR-4324, miR-7, miR-379, miR-543, etc. Among these micro RNAs, qRT-PCR results indicated that miR4324 is the only one that is dysregulated in ESCC tissues (Figure 1E). Figure $1 \mathrm{~F}-\mathrm{G}$ indicates that the downregulation of miR-4324 exists in $88 \%$ cases of ESCC tissues $\left({ }^{* *} p<0.01\right)$. Interestingly, we identified that the tumoral miR-4324 expression was negatively correlated with FAK levels in the collected 50 ESCC tissues $\left(\mathrm{R}^{2}=0.8236\right.$, Figure 1H). Similar results were acquired in cultured cancer cells, which reveals that miR-4324 is significantly downregulated in both TE-1 and Eca109 cells when compared with that in HECC cells (Figure 1I, ${ }^{* *} p<0.01$ ).

Subsequently, we explored the direct regulatory relationships between miR-4324 and FAK. Bioinformatic analysis reveals one potential miR-4324 binding site in the $3^{\prime}$ UTR (untranslated regions) of FAK mRNA (988-1008 amino acid,
Figure 2A). Dual-Luciferase reporter assay system with plasmids containing WT (pGL3-FAK-WT) or mutated (pGL3FAK-MT) FAK mRNA 3'UTR was employed to validate the specific and direct binding between miR-4324 and FAK mRNA. The results in Figure 2B indicated that overexpression of miR-4324 mimic dramatically reduced the luciferase reporter activity of WT but not mutated pGL3-FAK system. Besides, the intracellular FAK significantly decreased after miR-4324 mimic transfection in both mRNA (Figure 2C, ${ }^{* *} p<0.01$ ) and protein levels (Figure 2D).

\section{miR-4324 impairs ESCC cell invasion and migration in vitro}

The wound healing and trans-well assays were performed for evaluating the effects of miR-4324 and FAK on ESCC cell invasion and migration. Figure $3 \mathrm{~A}-\mathrm{B}$ reveals that both ESCC cells with miR-4324 mimic transfection exhibited significantly weaker motility than cells in WT and empty vehicle transfected $(\mathrm{NC})$ groups $(* * p<0.01)$. Consistently, the results of trans-well experiments demonstrated that miR-4324 mimic significantly impaired the migration activity and invasion potential in both TE-1 and Eca109 cells $(* * p<0.01)$ (Figure $3 \mathrm{C}-\mathrm{D})$. The most important is that the regulating roles of miR-4324 on cell invasion and migration can be effectively attenuated by overexpressing FAK in both ESCC cells (Figure 3A-D). 


\section{The EMT phenotype of ESCC cells is regulated by miR-4324/FAK axis}

As shown in Figure 3E, miR-4324 mimic increased the E-cadherin expression but attenuated the $\mathrm{N}$-cadherin and Vimentin expression in both ESCC cells. Likewise, these alterations caused by miR-4324 mimic was remarkably prevented by the co-transfection of FAK. As the downregulation of E-cadherin and upregulation of $\mathrm{N}$-cadherin \& Vimentin are principal characterizations of cellular EMT, ${ }^{12}$ these results indicate that miR-4324 mimic can significantly prevent the EMT process in ESCC cells, and the downregulation of FAK is involved in this EMT preventing effect.

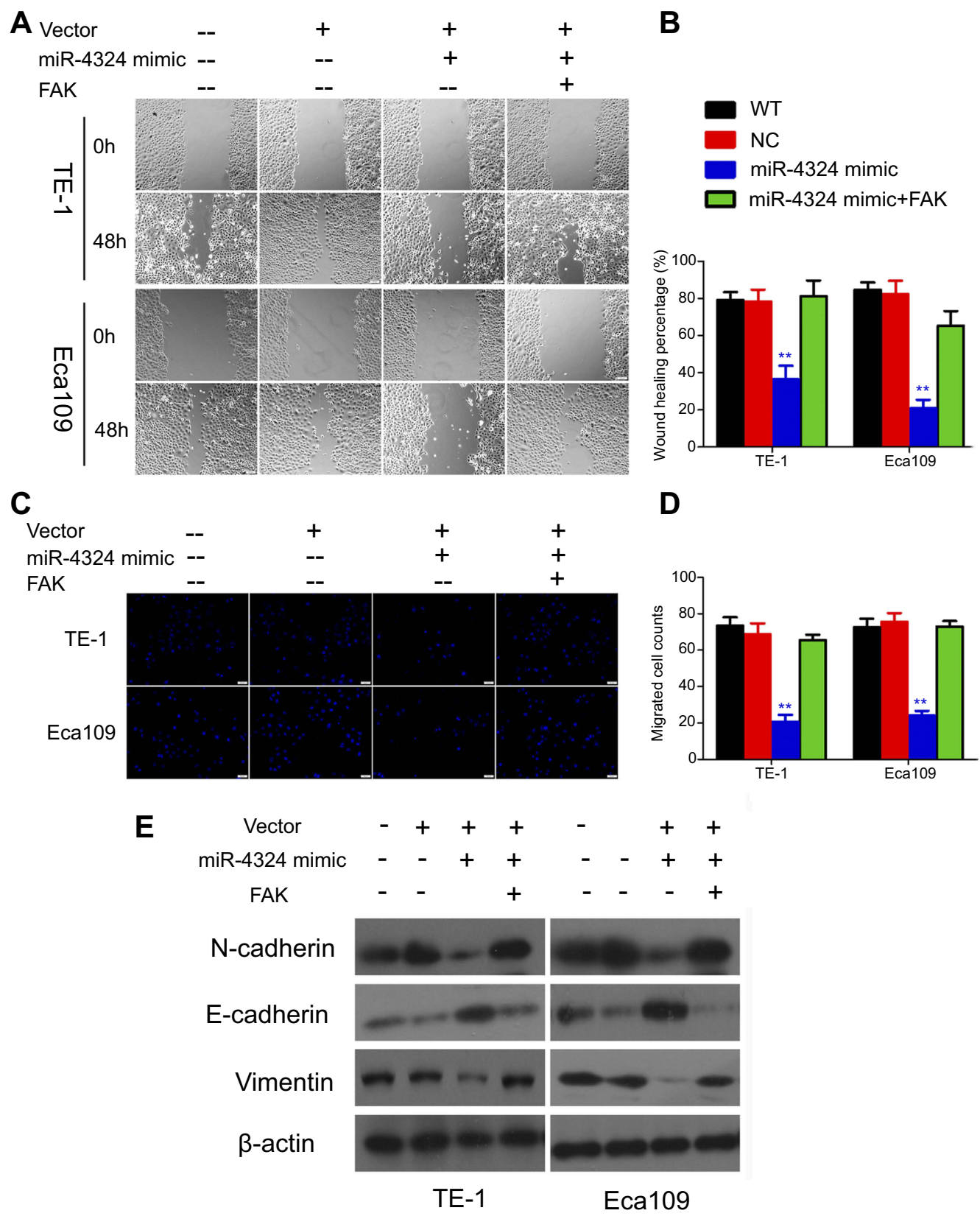

Figure 3 The functional significance of miR-4324 and FAK in ESCC cells. (A) Wound healing assays comparing the invasion potential of WT, mock-vehicle (NC) and miR4324/FAK transfected ESCC cells (magnification times: 100x, Scale bars: $100 \mu \mathrm{m}$ ). (B) Statistical analysis for the wound healing percentage of ESCC cells in different groups. (C) Trans-well assessment comparing the migration ability of both ESCC cells transfected with miR-4324/FAK in comparison with WT and mock-vehicle (NC) cells (magnification times: 100x, Scale bars: $20 \mu \mathrm{m}$ ). (D) Statistical analysis for the migrated cells per 100x microscopic field in different groups of Trans-well assays. (E) Relative expression of EMT markers in different groups of ESCC cells by WB analysis. Data are shown as mean $\pm S D(n=3),{ }^{* *} p<0.01$.

Abbreviations: WT, wild type; ESCC, esophageal squamous cell carcinoma; FAK, focal adhesion kinase; EMT, epithelial-mesenchymal transition; WB, Western Blotting; SD, standard deviation. 


\section{Clinical implication of miR-4324/FAK axis in ESCC patients}

In order to better understand the biological function and clinical implication of miR-4324 and FAK, 50 ESCC patients ranged from 44 to 71 years were enrolled in this study. The relative expression of miR-4324 and FAK in resected ESCC specimens were measured by qRT-PCR with the results showing in Figure 1. Statistical analysis (Table 1) reveals that low tumoral miR-4324 remarkably correlates with poor cell differentiation $(* p=0.015)$, tumor size $(* * p=0.007)$ and invasion $(* * p=0.007)$, as well as numbers of metastatic lymph nodes $(* * p=0.008)$, and high FAK was correlated with these clinic-pathological characterizations with the $p$-values of, respectively, 0.008, 0.004, 0.022 and 0.031. Subsequently, the Kaplan-Meier method was employed to evaluate the implication of miR-4324/FAK on the overall survival of the above mentioned 50 ESCC patients by a retrospective cohort study. As we can see, patients with higher tumoral miR-4324 (Figure 4A) or lower FAK (Figure 4B) (Red curve) lived longer than their counterparts (Green curve), respectively.

Table I Relationship between miR-4374/FAK expression and clinical pathologic parameters

\begin{tabular}{|c|c|c|c|c|c|c|c|}
\hline \multirow[t]{2}{*}{ Variables } & \multirow[t]{2}{*}{$\mathbf{N}=\mathbf{5 0}$} & \multicolumn{2}{|c|}{ miR-4374 expression } & \multirow[t]{2}{*}{$p$-value } & \multicolumn{2}{|c|}{ FAK expression } & \multirow[t]{2}{*}{$p$-value } \\
\hline & & $\operatorname{High}(n=2 I)$ & $\operatorname{Low}(n=29)$ & & $\operatorname{High}(n=32)$ & $\operatorname{Low}(n=18)$ & \\
\hline Age(years) & & & & 0.661 & & & 0.962 \\
\hline$\leq \mathbf{5 0}$ & 22 & 10 & 12 & & 14 & 8 & \\
\hline$>50$ & 28 & 11 & 17 & & 18 & 10 & \\
\hline Gender & & & & 0.634 & & & 0.793 \\
\hline Male & 29 & 13 & 16 & & 19 & 10 & \\
\hline Female & 21 & 8 & 13 & & 13 & 8 & \\
\hline Tumor diameter & & & & $0.007 * *$ & & & $0.004 * *$ \\
\hline$\leq 2 \mathrm{~cm}$ & 20 & 13 & 7 & & 8 & 12 & \\
\hline$>2 \mathrm{~cm}$ & 30 & 8 & 22 & & 24 & 6 & \\
\hline Cell differentiation & & & & $0.015^{*}$ & & & $0.008 *$ \\
\hline Poor & 29 & 8 & 21 & & 23 & 6 & \\
\hline Well to moderate & 21 & 13 & 8 & & 9 & 12 & \\
\hline Tumor invasion $(T)$ & & & & $0.007 * *$ & & & $0.022 *$ \\
\hline $\mathrm{TI} / \mathrm{T} 2$ & 20 & 13 & 7 & & 9 & 11 & \\
\hline T3/T4 & 30 & 8 & 22 & & 23 & 7 & \\
\hline Nodal status (N) & & & & $0.008 *$ & & & $0.031 *$ \\
\hline No I & 18 & 12 & 6 & & 8 & 10 & \\
\hline N2 & 32 & 9 & 23 & & 24 & 8 & \\
\hline
\end{tabular}

Notes: $*_{p}<0.05, * * p<0.01$

High: Relative expression FAK $\geq 5$ miR-4324 $\geq 2$

Low: Relative expression FAK $<5$ miR-4324<2

A

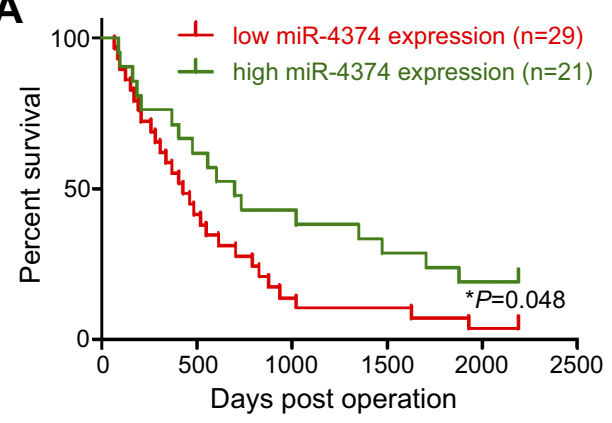

B

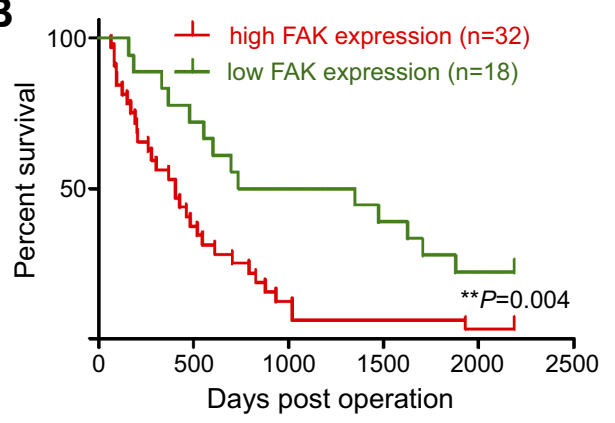

Figure 4 (A) Kaplan-Meier curve of prognosis of ESCC patients with high and low miR-4324 expression. (B) Kaplan-Meier curve of prognosis of ESCC patients with high and low FAK expression.

Abbreviations: ESCC, esophageal squamous cell carcinoma; FAK, focal adhesion kinase. 


\section{Discussion and conclusions}

Despite significant advances in disease treating and diagnosing, ESCC still owns a dismal 5-year survival rate of merely $19 \%$ in the United States according to a latest statistical reports released by the ACS, ${ }^{2}$ the fact of which calls for the identification of potential effective therapeutic and diagnostic targets. Presently, aberrant expression of FAK has been reported in a variety of malignancies. The abnormal expression, together with its well-established tumorigenic activities, highlights FAK as an attractive diagnostic and therapeutic target in curing cancers. ${ }^{31}$ Vassiliki Kostourou et al's study found that forced expression of FAK in endothelial cells enhances the angiogenesis of melanoma and lung carcinoma in mouse transplanted tumor models. ${ }^{32}$ Mengmeng $G u$ et al's study demonstrates that as a new FAK regulator, the p53-inducible gene 3 (PIG3) increased cancer metastasis, which could be considered as a novel and efficient prognostic biomarker and therapeutic target in treating lung adenocarcinoma metastasis. ${ }^{33}$ Stefanie Tiede and his colleagues found that repressing FAK activity by a FAK inhibitor (BI 853,520) significantly inhibits breast tumor cell proliferation and invasion in both in and ex vivo experiments. ${ }^{34}$ However, the roles of FAK on the initiation and progression of ESCC was rarely reported. Herein, for the first time we identified that the expression of FAK is significantly upregulated in both ESCC tissues \& cells (Figure 1). Therefore, relevant experiments were conducted to find out potential mechanisms of FAK regulation in ESCC.

MicroRNA is an emerging regulator with remarkable variety of biological function, contributing to the initiation and progression of various malignancies. ${ }^{35}$ It has been reported that FAK can be regulated by various micro RNAs in previous studies. ${ }^{27-30}$ However, miR-4324 is the only one significantly dysregulated in ESCC tissues among the 12 micro RNAs (Figure 1E). Interestingly, we found that the expression of FAK and miR-4324 was negatively correlated (Figure 1H). The intracellular levels of FAK experienced a significant decrease when ESCC cells were transfected with miR-4324 mimic (Figure 2), the results of which matched bioinformatics analysis predicting one potential miR-4324 binding site in the $3^{\prime} \mathrm{UTR}$ of FAK mRNA. What is more, miR-4324 overexpression impaired the migration activity and invasion potential in ESCC cells, which can be remarkably attenuated by the co-transfection of FAK (Figure 3 A-D). It seems that although one individual gene might be regulated by a variety of miRNAs, and one miRNA may regulate numerous targets, ${ }^{35}$ FAK is the most important among miR-4324 targets playing crucial roles in the tumorigenesis and development of ESCC.

It is generally considered that FAK is involved in the regulation of EMT, which is a pivotal step during cancer metastasis. ${ }^{11,31}$ During the process of EMT, non-invasive cancer cells lose their epithelial phenotype and acquire invasiveness, characterized by the reduced expression of E-cadherin and upregulation of N-Cadherin \& Vimentin. ${ }^{36}$ In this study, we identified that miR-4324 mimic remarkably downregulated EMT phenotype, which can also be prevented by FAK. The results of which may explain the underlying mechanisms of metastasis regulating functions of miR-4324/ FAK axis. Besides, the vital functions of miR-4324/FAK axis were verified by statistically analyzing the relevance between their tumoral expression and patient-clinic-pathological characteristics, with the results demonstrating that low miR-4324 and high FAK expression are, respectively, associated with poor cell differentiation, tumor size and invasion, as well as numbers of metastatic lymph nodes. Besides, survival analysis indicates that patients with higher tumoral miR-4324 levels and lower FAK levels, respectively, lived longer than their counterparts (Figure 4). All these results demonstrated that miR-4324-FAK axis could be a promising prognostic biomarker and potential therapeutic target for ESCC, which deserves further investigation in the clinic.

\section{Acknowledgments}

This study was supported by the Fund of Clinical Science and Technology of Wuxi (Q201620), Fund of Science and Technology of Yixing (2016-9).

\section{Disclosure}

The authors report no conflicts of interest in this work.

\section{References}

1. Ferlay J, Colombet M, Soerjomataram I, et al. Estimating the global cancer incidence and mortality in 2018: GLOBOCAN sources and methods. Int J Cancer. 2018. doi:10.1002/ijc.31937

2. Siegel RL, Miller KD, Jemal A. Cancer statistics, 2019. CA Cancer J Clin. 2019;69(1):7-34. doi:10.3322/caac.21551

3. Chen W, Zheng R, Baade PD, et al. Cancer Statistics in China, 2015. CA Cancer J Clin. 2016;66(2):115-132. doi:10.3322/caac.21338

4. Palladino-Davis AG, Mendez BM, Fisichella PM, Davis CS. Dietary habits and esophageal cancer. Dis Esophagus. 2015;28(1):59-67. doi: $10.1111 /$ dote. 12097

5. Kashima H, Noma K, Ohara T, et al. Cancer-associated fibroblasts (CAFs) promote the lymph node metastasis of esophageal squamous cell carcinoma. Int $J$ Cancer. 2019;144(4):828-840. doi:10.1002/ ijc. 31953 
6. Du D, Song T, Dai H, et al. Stereotactic body radiation therapy and thymosin alpha-1-induced anti-tumor effects in heavily pretreated, metastatic esophageal squamous cell carcinoma patients. Oncoimmunology. 2018;7(8):e1450128. doi:10.1080/2162402X.2018.1490854

7. Chen M, Xia Y, Tan Y, Jiang G, Jin H, Chen Y. Downregulation of microRNA-370 in esophageal squamous-cell carcinoma is associated with cancer progression and promotes cancer cell proliferation via upregulating PIN1. Gene. 2018;661:68-77. doi:10.1016/j.gene.2018.03.090

8. Ma Q, Liu W, Jia R, et al. Alcohol and survival in ESCC: prediagnosis alcohol consumption and postoperative survival in lymph node-negative esophageal carcinoma patients. Oncotarget. 2016;7 (25):38857-38863. doi:10.18632/oncotarget.8754

9. Hirano $\mathrm{H}$, Boku N. The current status of multimodality treatment for unresectable locally advanced esophageal squamous cell carcinoma. Asia Pac J Clin Oncol. 2018. doi:10.1111/ajco.12995

10. Kono K, Mimura K, Yamada R, et al. Current status of cancer immunotherapy for esophageal squamous cell carcinoma. Esophagus. 2018;15(1):1-9. doi:10.1007/s10388-017-0596-2

11. Foroni C, Broggini M, Generali D, Damia G. Epithelialmesenchymal transition and breast cancer: role, molecular mechanisms and clinical impact. Cancer Treat Rev. 2012;38(6):689-697. doi:10.1016/j.ctrv.2011.11.001

12. Nieto MA. The snail superfamily of zinc-finger transcription factors. Nat Rev Mol Cell Biol. 2002;3(3):155-166. doi:10.1038/nrm757

13. Mitra SK, Hanson DA, Schlaepfer DD. Focal adhesion kinase: in command and control of cell motility. Nat Rev Mol Cell Biol. 2005;6 (1):56-68. doi:10.1038/nrm1549

14. Zhang PF, Li KS, Shen YH, et al. Galectin-1 induces hepatocellular carcinoma EMT and sorafenib resistance by activating FAK/PI3K/ AKT signaling. Cell Death Dis. 2016;7:e2201. doi:10.1038/ cddis. 2015.324

15. Xu CY, Liu SQ, Qin MB, et al. SphK1 modulates cell migration and EMT-related marker expression by regulating the expression of p-FAK in colorectal cancer cells. Int $J$ Mol Med. 2017;39 (5):1277-1284. doi:10.3892/ijmm.2017.2921

16. Tian L, Cao J, Ji Q, et al. The downregulation of miR-3173 in B-cell acute lymphoblastic leukaemia promotes cell invasion via PTK2. Biochem Biophys Res Commun. 2017;494(3-4):569-574. doi:10.1016/j.bbrc.2017.10.013

17. Watermann DO, Gabriel B, Jager M, et al. Specific induction of pp125 focal adhesion kinase in human breast cancer. $\mathrm{Br} J$ Cancer. 2005;93(6):694-698. doi:10.1038/sj.bjc.6602744

18. Joseph NA, Chiou SH, Lung Z, et al. The role of HGF-MET pathway and CCDC66 cirRNA expression in EGFR resistance and epithelial-to-mesenchymal transition of lung adenocarcinoma cells. J Hematol Oncol. 2018;11(1):74. doi:10.1186/s13045-018-0557-9

19. Bartel DP. MicroRNAs: genomics, biogenesis, mechanism, and function. Cell. 2004;116(2):281-297.

20. Szymczyk A, Macheta A, Podhorecka M. Abnormal microRNA expression in the course of hematological malignancies. Cancer Manag Res. 2018;10:4267-4277. doi:10.2147/CMAR.S174476

21. Treiber T, Treiber N, Meister G. Regulation of microRNA biogenesis and its crosstalk with other cellular pathways. Nat Rev Mol Cell Biol. 2018. doi:10.1038/s41580-018-0070-6

22. Cao XY, Zhang XX, Yang MW, et al. Aberrant upregulation of KLK10 promotes metastasis via enhancement of EMT and FAK/ SRC/ERK axis in PDAC. Biochem Biophys Res Commun. 2018;499 (3):584-593. doi:10.1016/j.bbrc.2018.03.194
23. Yin CM, Suen WC, Lin S, Wu X-M, Li G, Pan X-H. Dysregulation of both miR-140-3p and miR-140-5p in synovial fluid correlate with osteoarthritis severity. Bone Joint Res. 2017;6(11):612-618. doi:10.1302/2046-3758.611.BJR-2017-0090.R1

24. Wu C, Wan W, Zhu J, Jin H, Zhao T, Li H, Induction of potent apoptosis by an anti-CD20 aptamer via the crosslink of membrane CD20 on non-Hodgkin's lymphoma cells. RSC Adv. 2017;7:5158-5166. doi:10.1039/C6RA27154E

25. Zhu J, Wu C, Li H, et al. DACH1 inhibits the proliferation and invasion of lung adenocarcinoma through the downregulation of peroxiredoxin 3. Tumour Biol. 2016;37(7):9781-9788. doi:10.1007/ s13277-016-4811-x

26. Kabiri A, Esfandiari E, Hashemibeni B, Kazemi M, Mardani M, Esmaeili A. Effects of FGF-2 on human adipose tissue derived adult stem cells morphology and chondrogenesis enhancement in Transwell culture. Biochem Biophys Res Commun. 2012;424 (2):234-238. doi:10.1016/j.bbrc.2012.06.082

27. Jeong D, Ham J, Park S, et al. MicroRNA-7-5p mediates the signaling of hepatocyte growth factor to suppress oncogenes in the MCF-10A mammary epithelial cell. Sci Rep. 2017;7(1):15425. doi:10.1038/s41598-017-15846-z

28. Chen JS, Li HS, Huang JQ, et al. MicroRNA-379-5p inhibits tumor invasion and metastasis by targeting FAK/AKT signaling in hepatocellular carcinoma. Cancer Lett. 2016;375(1):73-83. doi:10.1016/j. canlet.2016.02.043

29. Bing L, Hong C, Li-Xin S, Wei G. MicroRNA-543 suppresses endometrial cancer oncogenicity via targeting FAK and TWIST1 expression. Arch Gynecol Obstet. 2014;290(3):533-541. doi:10.1007/s00404-014-3219-3

30. Song J, Kim D, Jin EJ. MicroRNA-488 suppresses cell migration through modulation of the focal adhesion activity during chondrogenic differentiation of chick limb mesenchymal cells. Cell Biol Int. 2011;35(2):179-185. doi:10.1042/CBI20100204

31. Genna A, Gil-Henn H. FAK family kinases: the Yin and Yang of cancer cell invasiveness. Mol Cell Oncol. 2018;5(4):e1449584. doi:10.1080/23723556.2018.1449584

32. Kostourou V, Lechertier T, Reynolds LE, et al. FAK-heterozygous mice display enhanced tumour angiogenesis. Nat Commun. 2013;4:2020. doi:10.1038/ncomms3020

33. Gu MM, Gao D, Yao PA, et al. p53-inducible gene 3 promotes cell migration and invasion by activating the FAK/Src pathway in lung adenocarcinoma. Cancer Sci. 2018. doi:10.1111/ cas. 13818

34. Tiede S, Meyer-Schaller N, Kalathur R, et al. The FAK inhibitor BI 853520 exerts anti-tumor effects in breast cancer. Oncogenesis. 2018;7(9):73. doi:10.1038/s41389-018-0083-1

35. He L, Hannon GJ. MicroRNAs: small RNAs with a big role in gene regulation. Nat Rev Genet. 2004;5(7):522-531. doi:10.1038/ nrg1379

36. Celia-Terrassa T, Bastian C, Liu D, et al. Hysteresis control of epithelial-mesenchymal transition dynamics conveys a distinct program with enhanced metastatic ability. Nat Commun. 2018;9(1):5005. doi:10.1038/s41467-018-07538-7 


\section{Supplementary material}
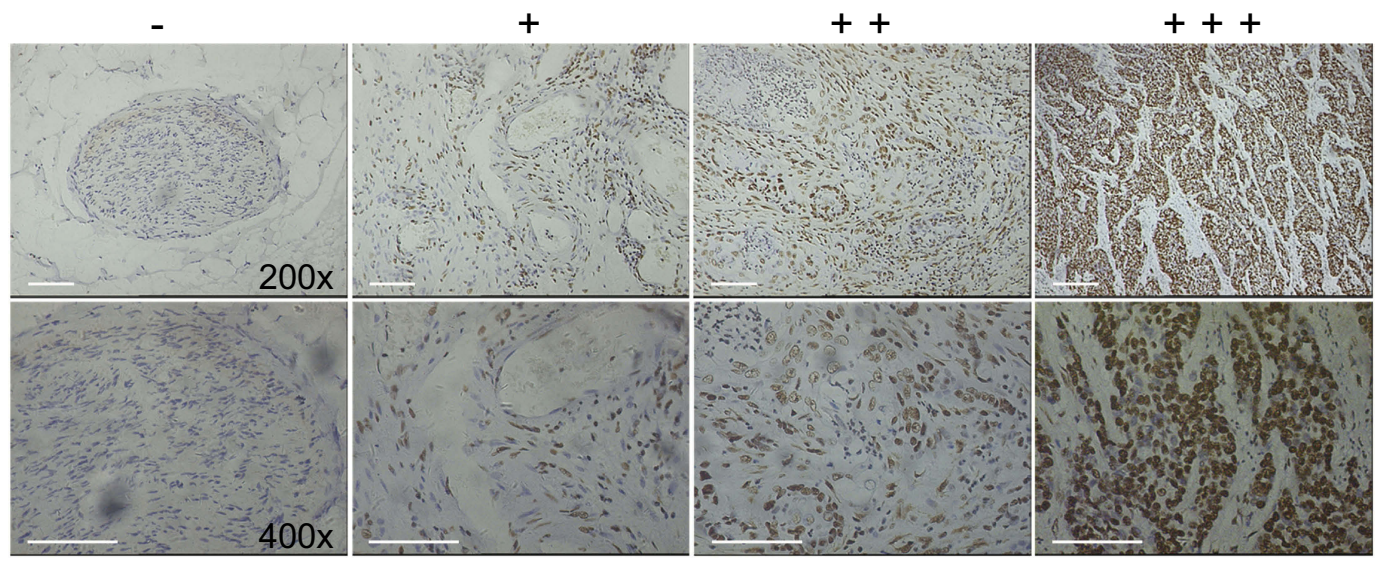

Figure SI Representative IHC staining for FAK staining in ESCC tissues, the expression level was scored as “-, +, ++, +++” (Magnification times: $200 \times$ and $400 \times$, Scale bars: $200 \mu$ m). Abbreviations: IHC, Immunohistochemistry; FAK, focal adhesion kinase; ESCC, esophageal squamous cell carcinoma.

Table SI Oligonucleotide sequences used in this study

\begin{tabular}{|l|l|}
\hline Oligonucleotide & Sequences \\
\hline FAK primer & Forward: 5'-GTGCTCTTGGTTCAAGCTGGAT-3' \\
GAPDH primer & $\begin{array}{l}\text { Reverse: 5'- ACTTGAGTGAAGTCAGCAAGAT-3' } \\
\text { Forward: 5'-TGCCACTCAGAAGACTGTGG-3' } \\
\text { Reverse: 5'- TTCAGCTCTGGGATGACCTT-3' }\end{array}$ \\
miRNA $\mathbf{4 3 2 4}$ & 5-CCCUGAGACCCUAACCUUAA-3'. \\
\hline
\end{tabular}

\section{Publish your work in this journal}

OncoTargets and Therapy is an international, peer-reviewed, open access journal focusing on the pathological basis of all cancers, potential targets for therapy and treatment protocols employed to improve the management of cancer patients. The journal also focuses on the impact of management programs and new therapeutic agents and protocols on patient perspectives such as quality of life, adherence and satisfaction. The manuscript management system is completely online and includes a very quick and fair peer-review system, which is all easy to use. Visit http://www.dovepress.com/ testimonials.php to read real quotes from published authors. 